\title{
Advances in patient-controlled analgesia: the role of fentanyl ITS
}

\author{
Ian Power \\ Jon G McCormack \\ Department of Anaesthesia, \\ Critical Care and Pain Medicine, \\ The University of Edinburgh, Royal \\ Infirmary, Edinburgh, UK
}

Correspondence: Jon McCormack Anaesthesia, Critical Care and Pain Medicine, The University of Edinburgh Royal Infirmary, Little France, Edinburgh EHI 6 4SA, UK

Tel +44 I 3 I 2423 I 36

Fax +44 । 3 | 2423।38

Email jon.mccormack@ed.ac.uk

\begin{abstract}
Effective pain relief is an essential component of a patient's peri-operative care package. Good analgesia has been shown to reduce the incidence of cardiovascular, respiratory and thrombo-embolic complications following surgery. Satisfactory analgesia facilitates early patient ambulation following surgery, which may reduce in-patient stay. Patient-controlled analgesia (PCA) systems are a well established standard therapy for acute post-operative pain; however some practical limitations limit their clinical utility. The fentanyl inotophoretic transdermal system (ITS) is a novel self-contained needle-free PCA device, which delivers boluses of fentanyl transdermally. This system has been shown to provide analgesia equivalent to conventional PCA modalities, with unique design features that may confer advantages to patients and staff, including facilitating patient mobilization in the post-operative phase. This review will discuss the technology of iontophoretic systems, the pharmacology of transdermal fentanyl delivery, and some practical implications of the fentanyl ITS.
\end{abstract}

Keywords: iontophoresis, transdermal, patient-controlled analgesia, fentanyl, post-operative pain

\section{Introduction}

Effective analgesia is a vital component of peri-operative care, yet survey results continue to suggest that the management of post-operative pain is often unsatisfactory (Apfelbaum et al 2003). Complications associated with moderate to severe post-operative pain are multi-systemic, and may have sequelae that by far outlast the acute peri-operative period. Intense sympathetic nervous system responses to painful stimuli will result in hypertension and may cause cardiac dysfunction or ischemia. Acute respiratory complications include hypoxia and pneumonia that may require critical care input and respiratory support. Severe or prolonged pain states trigger neurohumeral interactions that may induce neural network remodeling, promoting the development of neuropathic pain conditions or persisting complex pain syndromes (Kehler 1997; Dunwoody et al 2008). Non-physiological consequences of inadequately managed acute pain include restricted mobilization, which may limit the implementation of the pro-active co-ordinated multi-disciplinary approach to post-operative analgesia and ambulation that has been demonstrated to improve outcomes following major abdominal surgery (Fearon et al 2005). Poor patient mobilization may also lead to delayed hospital discharge, thus limiting the throughput of surgical units and potentially impacting on healthcare budgets (Fearon et al 2005).

Traditionally analgesia has been administered as intramuscular (im) or intravenous (iv) boluses of opioid analgesics. More recently, patient-controlled analgesia (PCA) systems have been established as a standard treatment for moderate to severe post-operative pain. In practice, PCA provides pain relief superior to the intermittent administration of bolus doses of opioids. Up to $30 \%$ of patients given im 
analgesia report severe post-operative pain, reducing to around $10 \%$ in patients treated post operatively with PCA systems (Dolin et al 2002).

This article will discuss recent developments in PCA technology that may further improve post-operative analgesic therapies, namely the fentanyl iontophoretic transdermal system (ITS). This system offers a number of advantages over conventional PCA systems. The needle-free method of drug delivery avoids the risk of complications from needle-stick injuries, reduces infection risk to both patients and staff and eliminates problems relating to drug extravasation. The pre-programmed electronics eradicate the potential for potentially fatal manual programming and dosing errors. Additionally, the compact size of the system may enable greater patient mobility following surgery. The fentanyl ITS has the potential to become a valuable option in the management of acute postoperative pain.

\section{Patient-controlled analgesia}

The worldwide drive to improve the treatment of post-operative pain over the last two decades has facilitated the establishment of dedicated multi-disciplinary acute pain services, supported by the development of acute pain management guidelines (Practice guidelines 2004). The concurrent popularization of PCA systems has resulted in marked advances in the management of acute post-operative pain (Macintyre 2001).

PCA is a well-established modality for the delivery of post-operative analgesia that has been shown to provide more effective pain relief and be preferred by patients over conventional parenteral methods of analgesic delivery (Dolin et al 2002). PCA allows patients to self-administer analgesics on demand according to their personal requirements for pain relief. Compared with boluses of iv or im analgesia, the smaller but more frequent boluses of opioids via PCA systems should reduce the risks of adverse events resulting from high peak plasma opioid levels. Similarly, patients can maintain a uniform level of analgesia and avoid breakthrough pain occurring from the inevitable "analgesic gaps" resulting from delays in the administration of analgesics that are prescribed on an "as-required" basis.

However, there are drawbacks associated with the technique that may limit the extent to which it can further enhance post-operative pain management. Drug delivery is invasive, requiring a needle for administration, introducing the risks of needle-stick injury and infection to the patient and hospital personnel. Staff education and equipment maintenance is mandatory to facilitate the safe delivery of PCA. Technical aspects of the infusion device must include safety features to prevent accidental overdose or deliberate opioid diversion, such as lockable syringe covers, anti-tamper controls and dedicated infusion sets. Patient ambulation is partly restricted by the continual presence of iv lines and the infusion pump mounted on a drip-stand. Risk management standards dictate that monitoring of analgesic efficacy, physiological parameters and physical performance of the pump requires assessment by trained individuals on a regular basis.

\section{Development of new devices}

The development of novel analgesics and sophisticated delivery techniques has the potential to improve current strategies for post-operative pain management. The fentanyl hydrochloride iontophoretic transdermal system (fentanyl ITS) is a novel PCA system that has been approved in Europe and North America for the management of acute, moderate to severe post-operative pain in hospitalized patients. The fentanyl ITS is an advanced pain management system that may address many of the above concerns of safety and convenience with existing PCA modalities through the use of a pre-programmed and disposable drug delivery system. As opposed to passive transdermal fentanyl patches, the use of iontophoretic technology allows for the self-administration of a precise dose of transdermal opioid with a pharmacokinetic profile that is appropriate for acute post-operative pain management.

The transdermal route of analgesic delivery offers a number of advantages over existing strategies for the systemic administration of analgesics. The requirements for the use of needles and venous access are eliminated, making analgesic administration less invasive, simpler, and more convenient. Like other parenteral routes, drug delivery via the transdermal route also bypasses first-pass hepatic metabolism and circumvents common barriers to the use of oral analgesics immediately following surgery, such as nausea and vomiting, and difficulty swallowing. However, conventional transdermal analgesic modalities rely on passive drug diffusion creating a skin depot of active drug, from which there may be prolonged and unpredictable absorption (Varvel et al 1989). Passive transdermal systems are also unable to administer individual, on-demand doses, and thus are inappropriate strategies for acute post-operative pain management.

\section{Overview of ITS}

The fentanyl ITS (IONSYS ${ }^{\mathrm{TM}}$, Janssen-Cilag NV, Beerse, Belgium) has recently been approved for clinical prescription in the UK. This credit card-sized needle-free pre-programmed 
system may be applied to the patient's chest or upper outer arm using an adhesive that covers the bottom of the drug component housing (Figure 1). The patient activates the system by pressing the recessed dosing button twice within 3 seconds. Upon activation, the fentanyl ITS administers a pre-programmed $40-\mu \mathrm{g}$ dose of fentanyl over a 10 -minute period. Additional dosing requests are prevented by the system during drug delivery, thus patients may self-administer up to 6 doses per hour. The fentanyl ITS operates for up to 24 hours or a maximum of 80 doses (whichever occurs first), after which it automatically shuts down. The system may then be removed and discarded, and a new system may be applied to a different skin site if additional analgesia is required.

The entire unit is self-contained within a compact plastic housing. The dosing status of the system is indicated by a small, light-emitting diode (LED) and audible beeps. Illumination of the red LED and a single beep indicate the beginning of each dose, and the LED remains on until delivery of a dose has completed. Between doses, the system automatically communicates the approximate number of doses delivered via a series of light flashes from the LED. The total dose count may also be displayed during drug delivery by pressing the dosing button once. This device address many of the limitations with traditional PCA devices, with regards to set-up and maintenance, patient restrictions on mobilization due to the iv lines and infusion pumps, and the labor demands of the regular checking of devices. However, the demands of patient safety monitoring of physiological parameters and analgesic efficacy are unchanged. Use of this device may facilitate earlier ambulation in keeping with the enhanced recovery after surgery (ERAS) concepts.

\section{lontophoresis}

In order to administer analgesic agents by the transdermal route, they must have certain characteristics, including a low molecular weight ( $<600 \mathrm{Da})$, appropriate solubility in water and oil, an optimal partition coefficient between the membrane and the solution, and a low melting point. Fentanyl has a low molecular weight of $286 \mathrm{~g} / \mathrm{mol}$, high lipophilicity (octanol-water partition coefficient of 717), and optimal skin flux (approximately 1000 times that of morphine) (Grond et al 2000). The physicochemical characteristics of fentanyl make it not only the optimal analgesic agent for transdermal administration, but an ideal agent for iontophoretic transdermal delivery, where it has been shown that iontophoretic administration can result in circulating plasma concentrations similar to those achieved by iv infusion using a low-intensity electric current (Sathyan et al 2005a).

\section{Dimensions : $75 \times 50 \times 8 \mathrm{~mm}$}
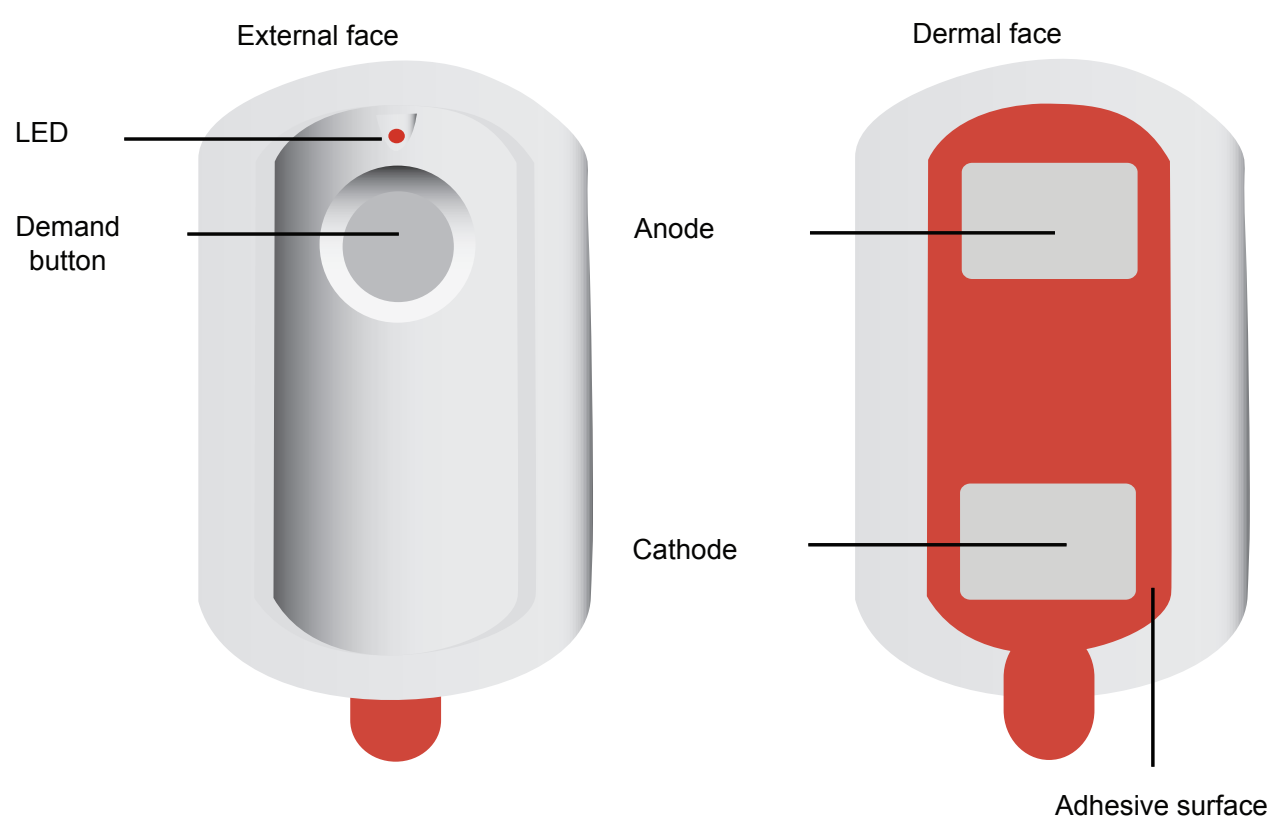

Figure I The fentanyl ITS. Image shows the exterior aspect, with the on/bolus button, and the indicator LED (light emitting diode). The electrodes are visible on the interior aspect, which contacts the patient's skin), and the self-adhesive surround. 
The fentanyl ITS significantly enhances the rate of transdermal fentanyl delivery relative to passive diffusion. Iontophoresis is the process by which an indiscernibly low intensity electric current $(170 \mu \mathrm{A})$ is used to drive ionized drug molecules actively by the process of electro-repulsion across the stratum corneum of the skin and into the systemic circulation. Electric current flows from the anode to the cathode, with the skin completing the circuit. Current flowing through the drugcontaining anode hydrogel reservoir drives positively charged fentanyl molecules into the skin via both electro-repulsion of similarly charged molecules and bulk fluid flow resulting from electro-osmosis (Figure 2). The flow of electric current may also increase skin permeability, which is reversible and not indicative of skin damage (Curdy et al 2001; Pikal et al 2001).

\section{Pharmacology and pharmacokinetics}

As the transdermal patch administers fentanyl continuously after application to the skin, it lacks the ability to deliver individual, on-demand doses. Across studies, the mean time to maximum fentanyl serum concentration $\left(t_{\max }\right)$ was shown to range from 12 to 48 hours using the fentanyl patch, depending upon both the delivery rate and the duration of administration (Grond et al 2000). In contrast, a single study demonstrated that iontophoretic delivery of fentanyl using the fentanyl ITS resulted in a $t_{\max }$ of approximately 39 minutes after the completion of delivery of a single dose (Sathyan et al 2005a). The decline in serum fentanyl concentrations following termination of treatment also occurs more rapidly with the fentanyl ITS than with the fentanyl patch, with an elimination half-life of 11 versus 13 to 25 hours (Grond et al 2000; Sathyan et al 2005a). These data suggest that fentanyl does not accumulate in a skin depot during drug delivery by the fentanyl ITS, in contrast to the gradual release of fentanyl into the systemic circulation after cessation of treatment with the transdermal fentanyl patch.

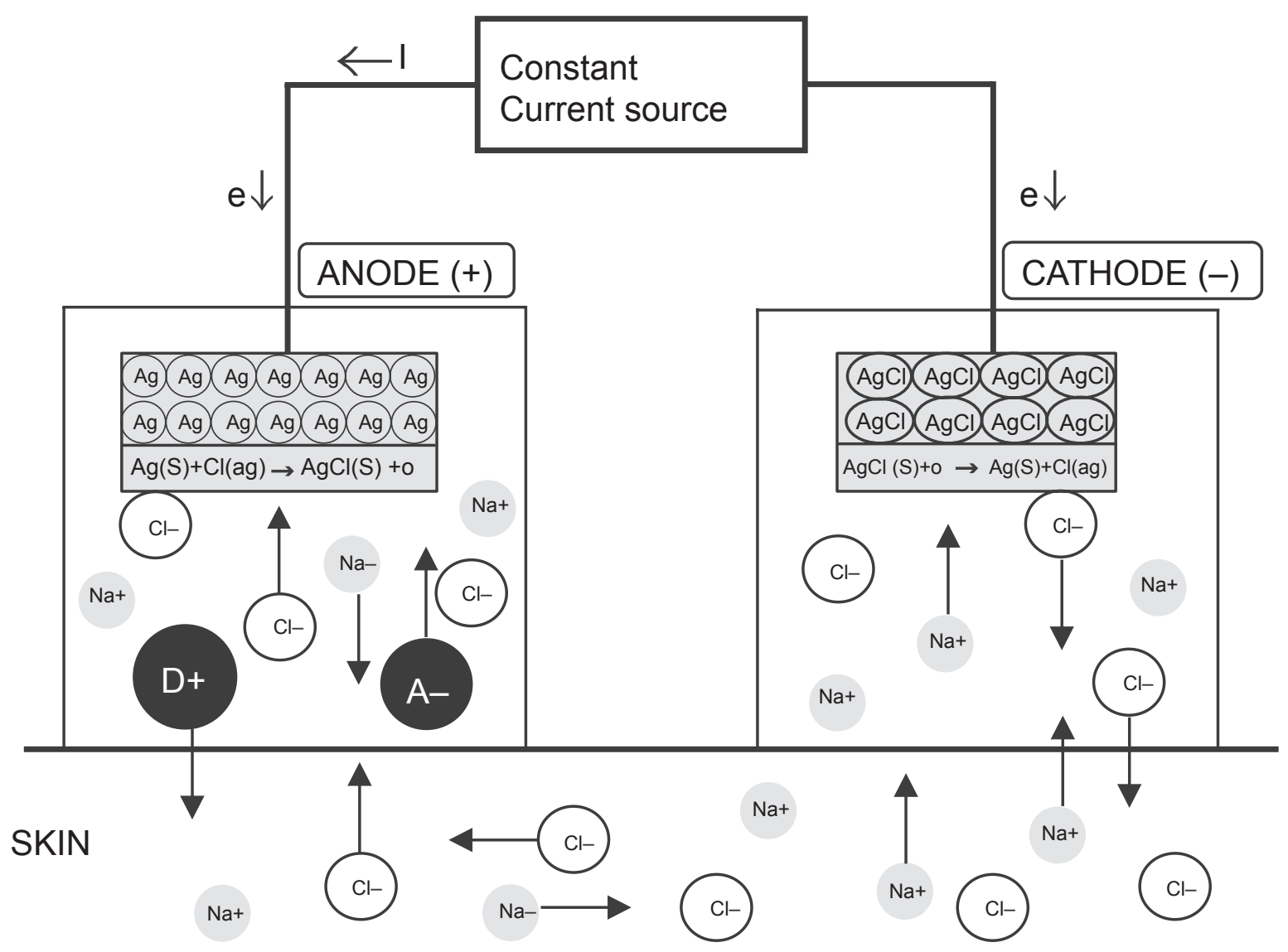

Figure 2 lontophoretic drug delivery. Schematic diagram of iontophoretic drug delivery (Ag/AgCl electrode system). lontophoretic devices typically utilize anodal transport, in which the ionizable drug $\left(D^{+}\right)$and its counter-ion $\left(A^{-}\right)$are contained within the anodal compartment, to administer therapeutics transdermally. $A$ low-intensity electric current (e) repels ionized drug molecules $\left(D^{+}\right)$from the anodal drug reservoir, driving them across the epidermis and into the subdermal tissue, where they are absorbed into the systemic circulation. Simultaneously, chloride ions $\left(\mathrm{Cl}^{-}\right)$are repelled from the cathode hydrogel reservoir. To maintain electroneutrality, oppositely charged ions, primarily $\mathrm{Cl}^{-}$and $\mathrm{Na}^{+}$, move into the anode and cathode reservoirs, respectively. Reproduced with permission from Kalia YN, Naik A, Garrison J, Guy RH. 2004. lontophoretic drug delivery. Adv Drug Deliv Rev, 56:619-58. Copyright (C) 2004 Elsevier. 
The fentanyl ITS delivers controlled doses of fentanyl only after activation of its electronic circuitry. Minimal passive diffusion of fentanyl was found to occur from an inactivated fentanyl ITS, resulting in clinically insignificant drug absorption into the serum (Grond et al 2000). In experimental conditions, iontophoresis strongly increased transdermal permeation of the drugs as compared to passive diffusion in a charge-dependent manner. The maximal plasma concentrations were $0,0.76$ and $1.59 \mathrm{ng} / \mathrm{mL}$ with driving currents of 0,1 and $2 \mathrm{~mA}$, respectively $(\mathrm{p}=0.010)$. Mean time to detection of plasma fentanyl following iontophoretic administration was 19 to 33 minutes (Ashburn et al 1995).

When comparing fentanyl ITS with iv administered fentanyl, serum concentrations differ significantly at 10 minutes after the initial dose is administered: $0.1 \mathrm{ng} / \mathrm{mL}$ for fentanyl ITS versus $0.7 \mathrm{ng} / \mathrm{mL}$ for iv fentanyl. However, as iontophoretic absorption increases in a time-dependent manner over the first 10 hours of dosing, the pharmacokinetic parameters of fentanyl ITS become directly comparable to those of iv fentanyl after 24 hours: maximum plasma concentrations are 1.37 and $1.82 \mathrm{ng} / \mathrm{mL}$, time to maximum concentration 35 and 39 minutes, and area under the concentration-time curve at 24 hours 1.23 and $1.34 \mathrm{ng} . \mathrm{h} / \mathrm{mL}$ for fentanyl ITS and intravenous fentanyl, respectively (Herndon 2007). The mean serum terminal half-life $\left(t_{1 / 2}\right)$ after cessation of fentanyl ITS treatment is 11 hours, with a slope of the terminal serum concentration decline similar to that observed after iv fentanyl infusion of 13 hours (Sathyan et al 2005a), supporting the hypothesis that fentanyl ITS drug delivery does not cause an accumulation of fentanyl in a skin depot.

The 40- $\mu \mathrm{g}$ dose of fentanyl delivered by the fentanyl ITS was selected based on the results of a dose-finding study that evaluated 3 fentanyl doses $(20 \mu \mathrm{g}, 40 \mu \mathrm{g}$ and $60 \mu \mathrm{g})$, which found this dose to optimize analgesic efficacy with an acceptable side effect profile (Camu et al 1998). The higher dose $(60 \mu \mathrm{g})$ increased respiratory depression, while the lower dose $(20 \mu \mathrm{g})$ did not provide adequate analgesia.

Clinical studies have investigated the impact of various parameters on the pharmacokinetic profile of fentanyl delivered by the system. The amount of fentanyl absorbed by patients was found to be directly proportional to the magnitude of current applied by the system, with a $170 \mu \mathrm{A}$ current producing absorption of approximately $40 \mu \mathrm{g}$ fentanyl and a coefficient of variation similar to that of iv fentanyl infusion (22\% and $23 \%$, respectively) (Sathyan et al 2005a). A separate study found that fentanyl absorption increases with time; in the first hour of treatment approximately $40 \%$ of each $40 \mu \mathrm{g}$ bolus dose of fentanyl is absorbed; however after 10 hours of ITS therapy almost $100 \%$ of each $40 \mu \mathrm{g}$ bolus is absorbed (Sathyan et al 2005b). Although the reasons for the increase in fentanyl absorption over time are not entirely clear, it may result from the reversible alterations in the electrical conduction properties of skin that occur during exposure to electric current from the system (Curdy et al 2001). Absorption of fentanyl was not affected, however, by variations in the frequency of dosing or patient characteristics, such as age, gender, ethnicity, and weight (Sathyan et al 2005b).

\section{Efficacy studies}

The fentanyl ITS has been evaluated in the treatment of acute, moderate-to-severe post-operative pain in adult patients undergoing orthopedic, abdominal, or thoracic surgery in several multi-center studies with both placebo and active comparators.

\section{Fentanyl ITS versus placebo}

Initial assessments in a number of randomized, placebocontrolled clinical trials found the fentanyl ITS to provide pain relief superior to placebo (Chelly et al 2004; Viscusi et al 2006). Patients were treated for up to 24 hours with the fentanyl ITS (40 $\mu \mathrm{g}$ fentanyl $/ 10$ minutes; maximum, $240 \mu \mathrm{g} /$ hour) or a placebo system containing modified electronic circuitry to prevent iontophoretic dosing. Compared with the placebo device in almost 500 patients, $29 \%$ versus $60 \%$ of subjects reported inadequate analgesia when using fentanyl ITS. Mean pain intensity scores were lower (3.5 versus 5.4$)$ and both patients and investigators reported the fentanyl ITS as a good or excellent method of pain control, in over $70 \%$ of cases (Viscusi et al 2006).

\section{Fentanyl ITS vs transdermal fentanyl}

A commercially available fentanyl patch uses transdermal fentanyl delivery for the effective management of chronic pain (Muijsers and Wagstaff 2001). The patch continuously delivers fentanyl through the skin via passive diffusion from a polymer matrix. Fentanyl delivery by the transdermal patch is characterized by a slow rate of drug absorption and sustained serum concentrations after patch removal, ideal for the management of chronic pain conditions.

\section{Fentanyl ITS versus iv opioids}

A prospective multi-center randomized controlled trial comparing the fentanyl ITS to iv PCA morphine in 650 patients demonstrated identical patient global assessment of pain control, with over $80 \%$ of patients reporting 
good or excellent pain relief in both treatment arms following major abdominal or orthopedic surgery, with similar pain intensity scores and side-effects reported in both groups (Grond et al 2007). Similar satisfaction and pain intensity score reporting was repeated in further active-comparator studies (Table 1) (Viscusi et al 2004; Hartrick et al 2006; Minkowitz et al 2007). There were no significant differences in the rate of withdrawal of patients from these studies due to inadequate analgesia (around 10\%-15\% in both iv PCA and ITS groups). It should be borne in mind that these studies were all open-label and without blinding, and as such, subject withdrawal due to patient, staff or investigator bias cannot be excluded.

\section{Safety and tolerability}

The fentanyl ITS has a favorable safety profile, particularly when considering potential programming or dosing errors. It is effectively pre-loaded with active drug, eliminating the requirement for point-of-care preparation of parenteral opioids, which has repeatedly been shown to be risk prone and has previously been a contributory factor in patient mortality, with human error accounting for around one-quarter of adverse incidents related to PCA use (Vicente et al 2003; Chelly 2005). As the fentanyl ITS is pre-programmed neither dose nor lockout period may be modified, eliminating the risk of manual errors related to dose calculations, and infusion device programming. However, these properties remove the possibility for dosing adjustments, which may be potentially limiting for patients with unique or increased opioid needs. Patient safety is further enhanced by the "lock-out" period during analgesic delivery to prevent overmedication from excessive dosing, which is, of course, a feature of all PCA systems.

\section{Systemic complications}

Adverse events reported when using the fentanyl ITS are comparable to that of iv PCA morphine, and indeed similar to that of all opioids whichever administration route is employed (Viscusi et al 2004, 2006; Hartrick et al 2006; Grond et al 2007; Minkowitz et al 2007). As with iv PCA morphine, nausea was the commonest reported adverse-event in around $40 \%$ to $50 \%$ of subjects in an active-comparator phase III trial (Tramer and Walder 1999). Less frequently, vomiting, pruritis and headache have a similar reporting rate between both analgesic modalities. Adverse event data are summarized in Table 2. Common to all systemic opioid drug prescriptions, the most potentially serious adverse event is respiratory depression, with an incidence of around $0.5 \%$ to $2 \%$ with iv PCA systems (Sidebotham et al 1997 ; Anghelescu et al 2005). While the risk must be recognized and appropriate monitoring mechanisms be in place, there were no events of clinically important respiratory depression, defined as $<8$ breaths/minute for 1 minute and excessive sedation, reported in any of the 1288 patients studied during fentanyl ITS therapy (Chelly 2005). As with all parenteral opioid administration, careful patient selection should reduce the risk of serious adverse events occurring.

Table I Summary of efficacy data

\begin{tabular}{|c|c|c|c|c|c|c|c|c|}
\hline \multirow[t]{3}{*}{ Author } & \multicolumn{2}{|c|}{ Viscusi 2004} & \multicolumn{2}{|c|}{ Hartrick 2006} & \multicolumn{2}{|c|}{ Minkowitz 2007} & \multicolumn{2}{|c|}{ Grond 2007} \\
\hline & ITS & PCA & ITS & PCA & ITS & PCA & ITS & PCA \\
\hline & $n=316$ & $n=320$ & $n=395$ & $n=404$ & $n=252$ & $n=254$ & $n=325$ & $n=335$ \\
\hline \multicolumn{9}{|l|}{ Patient Global } \\
\hline \multicolumn{9}{|l|}{ Assessment } \\
\hline Excellent/Good & $74 \%$ & $77 \%$ & $83 \%$ & $82 \%$ & $85 \%$ & $84 \%$ & $86 \%$ & $88 \%$ \\
\hline Fair & $12 \%$ & $13 \%$ & $9 \%$ & $9 \%$ & $11 \%$ & $12 \%$ & $8 \%$ & $9 \%$ \\
\hline Poor & $13 \%$ & $8 \%$ & $7 \%$ & $7 \%$ & $4 \%$ & $2 \%$ & $4 \%$ & $3 \%$ \\
\hline IGA success & $79 \%$ & $82 \%$ & $86 \%$ & $83 \%$ & $89 \%$ & $89 \%$ & $91 \%$ & $90 \%$ \\
\hline $\begin{array}{l}\text { Last mean pain intensity } \\
\text { score }\end{array}$ & 33 & 31 & 3.0 & 3.0 & 3.0 & 2.9 & 2.5 & 2.4 \\
\hline \multicolumn{9}{|l|}{ Discontinuation } \\
\hline Inadequate analgesia & $15 \%$ & $10 \% *$ & $11 \%$ & $5 \% *$ & $9 \%$ & $3 \% *$ & $3 \%$ & $2 \%$ \\
\hline Adverse events & $6 \%$ & $6 \%$ & $3 \%$ & $6 \% *$ & $6 \%$ & $8 \%$ & $3 \%$ & $5 \%$ \\
\hline
\end{tabular}

Patient global assessment at 24 hours was the primary end-point in all four trials. Viscusi et al (2004) reported pain intensity on a Visual Analogue Scale where 0 mm represents no pain and $100 \mathrm{~mm}$ represents worst possible pain. The other studies report Numerical Rating Scale $(0-10)$ values. ${ }^{*} \mathrm{p}<0.05$.

Abbreviations: ITS, inotophoretic transdermal system; PCA, patient-controlled analgesia. 


\section{Local complications}

Iontophoresis has been shown to be well tolerated by the skin, and the sensations that occur as a result of current flow have typically been minor. Application site reactions have been reported in around $20 \%$ of subjects. These were generally mild and self-limiting, including erythema and itch beneath the electrodes which needed no intervention (Viscusi et al 2006). It is not clear, however, how many cases may have been of a more prolonged nature. Six subjects from a total of 1288 withdrew from the four active-comparator studies due to application side reactions.

\section{Patient perspectives}

The fentanyl ITS has been repeatedly shown to provide comparable post-operative analgesia with a similar adverse event profile to the accepted present standard of iv PCA morphine. It is important to note that the quality of analgesia has not been shown to be any better than PCA morphine; however, there are two key benefits worthy of discussion.

Firstly, analysis of the 'analgesic gaps' demonstrates superiority of ITS over PCA. An 'analgesic gap' is a time period where the patient requests analgesia, but for many potential reasons, analgesia is not delivered. These delays are apparent when analgesia is delivered by a nurse or physician administered bolus, and the analgesic gap exists during the time from initial perception of pain, until pain relief: during which time the staff must be alerted; perform pain assessment; prepare the appropriate analgesic to be administered (usually required two ward staff); then administer the analgesic. However, these analgesic gaps may also exist when using PCA systems. Pooled results from two of the active-comparator studies demonstrate a significantly lower rate of analgesic gaps when using ITS versus PCA ( $6 \%$ versus $12 \%$ ), and that the median time of each gap event was shorter (15 versus 20 minutes) (Panchal et al 2007).

Table 2 Pooled adverse event data from active-comparator studies (Viscusi et al 2005; Hartrick et al 2006; Grond et al 2007; Minkowitz et al 2007)

\begin{tabular}{lll}
\hline Adverse event & $\begin{array}{l}\text { Fentanyl ITS } \\
\mathbf{n}=\mathbf{I 2 8 8}\end{array}$ & $\begin{array}{l}\text { Morphine PCA } \\
\mathbf{n}=\mathbf{I 3} \mathbf{3}\end{array}$ \\
\hline Nausea & $39 \%$ & $43 \%$ \\
Application site reactions & $21 \%$ & $0 \%$ \\
Vomiting & $12 \%$ & $12 \%$ \\
Pruritis & $6 \%$ & $9 \%$ \\
Hypotension & $3 \%$ & $5 \%$ \\
\hline
\end{tabular}

Abbreviations: ITS, inotophoretic transdermal system; PCA, patient-controlled analgesia.
The commonest reason for an analgesic gap using the fentanyl ITS was device failure (reported in $4 \%$ subjects).

The second apparent advantage of ITS over PCA lies in the ability to ambulate patients following major surgery. National guidelines recommend that early post-operative mobilization should be an integral part of the peri-operative care package, which may facilitate reductions in the rate of post-operative thrombo-embolic events, pulmonary complications and length of in-patient stay (Walder et al 2001). Ambulation in the immediate post-operative phase requires optimization of three aspects: adequate analgesia; appropriate staff levels; freedom from connections to immobile equipment. These points have already been addressed in that fentanyl ITS has been shown to provide good or excellent analgesia in over $80 \%$ of patients, and there have been suggestions that these devices score more favorably compared to PCA and may be less demanding on labor resources from ward staff (Minkowitz et al 2007), saving an average of 68 minutes from time of prescription to analgesic delivery compared to iv PCA (Bonnet et al 2006). Subjective and objective perceptions of patient mobility have been assessed in the active-comparator trials (Hartrick et al 2006; Grond et al 2007; Minkowitz et al 2007) demonstrating around $95 \%$ of subjects using fentanyl ITS reporting no interference with ambulation, compared with $40 \%$ to $50 \%$ of subjects using morphine PCA apparatus.

A standard assessment tool for the utility of PCA devices in research studies is patient and investigator global assessment: in a placebo-controlled study of 484 participants, patient global assessment (PGA) rated the fentanyl ITS as excellent or good in $73 \%$, with the study investigators' global assessment (IGA) rated the ITS as excellent or good in $72 \%$; the system was rated as poor by PGA and IGA in $11 \%$ of uses (Viscusi et al 2006). In the same study, a patient satisfaction questionnaire demonstrated that the ITS was very convenient $(80 \%)$ and easy to use $(87 \%)$. A validated Ease of Care (EOC) questionnaire (Harding et al 2007) evaluated subjects' confidence, comfort and understanding of ITS over PCA devices in the active-comparator trials (Table 3) (Hartrick et al 2006; Grond et al 2007; Minkowitz et al 2007). Similarly, nurses and physiotherapists were asked complete EOC questionnaires to rate ITS and PCA systems with regards to time efficiency and interference with necessary activities related to patient care. On a six-point scale where 0 relates to "not at all" and 5 relates to "a great deal" with regards to time-consumption and interference with care activities, fentanyl ITS and morphine PCA respectively were 
Table 3 Pooled mean scores from EOC questionnaires in the active-comparator trials (Hartrick et al 2006; Grond et al 2007; Minkowitz et al 2007)

\begin{tabular}{lll}
\hline EOC Assessment Subscale & $\begin{array}{l}\text { Fentanyl ITS } \\
\mathbf{n = 9 4 2}\end{array}$ & $\begin{array}{l}\text { iv PCA } \\
\mathbf{n}=\mathbf{9 4 6}\end{array}$ \\
\hline Confidence & 4.51 & 4.15 \\
Movement & 4.86 & 3.83 \\
Comfort & 4.70 & 4.36 \\
Understanding & 4.21 & 3.9 \\
\hline
\end{tabular}

Abbreviations: EOC, ease of care; ITS, inotophoretic transdermal system; PCA, patient-controlled analgesia.

rated as 0.7 vs 1.2 by nurses and 0.5 vs 0.7 by physiotherapists $(\mathrm{p}<0.001)$ (Grond et al 2007).

Values represent the mean of a score on a validated six-point scale of 0 to 5 , where 0 relates to "not at all" and 5 relates to "a great deal". A higher value represents a more favorable outcome ( $\mathrm{p}<0.05$ for all values).

Alongside considerations of clinical evidence, the cost-effectiveness of medical interventions plays a sizeable role in healthcare decision-making. Cost evaluations of pain management must examine many direct and indirect expenses incurred by treatment. For example, the costs associated with iv PCA include those directly associated with the PCA pump (eg, costs related to purchase or rental, maintenance, and replacement) and consumable materials (eg, medication, iv tubing) in addition to labor costs (eg, training to use the device, set-up of the apparatus, loading of analgesic, programming the pump). The majority of the cost of treatment using the fentanyl ITS is associated with its purchase price. Labor costs would be expected to be minimal, as there is no requirement for programming or assembly of the system. Because the system is discarded after a single use, no resources would be required for maintenance or repair. While acknowledging that the pharmacoeconomics of using fentanyl ITS will be variable between different healthcare systems, it is possible to perform cost-effectiveness analysis based on current prices to the NHS in the UK. Exclusively considering raw materials, the fentanyl ITS costs around 10 times that of a standard PCA for 24 hours of therapy. However, it is difficult to specifically quantify cost savings in conjunction with potential reductions in labor intensity of ward staff and length of in-patient stay exclusively attributable to utilization of a fentanyl ITS system. One-way sensitivity analyses demonstrate very marginal financial implications of utilizing fentanyl ITS, with confidence intervals ranging between a cost saving of around $£ 30$ to a cost increment of $£ 20$ per patient use.

\section{Conclusion}

Pain management is an essential element of patient care and rehabilitation following surgery, as results of clinical studies have shown that effective pain control can reduce patient morbidity and its associated healthcare costs, in addition to minimizing patient anxiety and physical discomfort. However, PCA strategies that are commonly used to treat post-operative pain introduce some risk of potentially dangerous complications resulting from use of an invasive method of drug administration and the requirement for manual programming of a PCA pump. In addition, existing PCA devices may limit patient mobility and consume substantial resources dedicated to use and maintenance of the systems.

The fentanyl ITS offers an effective and safe alternative to traditional iv PCA systems. It has been shown to be as efficacious as PCA morphine, is well tolerated by patients and has a favorable safety profile. There are few limitations associated with use of the fentanyl ITS in post-operative pain management. One potential limitation is the inability to adjust the dosing parameters, which may impact pain management in patients with considerable opioid needs. In addition, the system is associated with limitations that are common to PCA in general. Patients must be able to understand instructions for operation of the system and sufficiently alert and physically able following surgery to be suitable candidates for self-management of post-operative pain. Patients who have impaired cognitive function, or restricted mobility as a function of neuro-muscular disease, severe arthritis or site of surgery (for example, upper limb surgery) may be unable to use the fentanyl ITS, as they would with traditional iv PCA systems. The authors' experience suggests that patients studied locally have no greater difficulties activating the fentanyl ITS rather than an iv PCA handset, though at present there are no objective data to support this.

The fentanyl ITS is an advanced pain management system that may address many of the concerns of safety and convenience with existing PCA modalities through the use of a pre-programmed and disposable drug delivery system. The application of iontophoretic technology allows for non-invasive opioid analgesic delivery with a pharmacokinetic profile appropriate for the relief of acute pain in the post-operative setting.

\section{Acknowledgment}

The authors would like to acknowledge input of Stephanie Dodds, Research Nurse, University of Edinburgh, for her information regarding practical aspects of the fentanyl ITS. 


\section{Disclosures}

The authors have no conflicts of interest to disclose.

\section{References}

Anghelescu DL, Burgoyne LL, Oakes LL, et al. 2005. The safety of patient-controlled analgesia by proxy in pediatric oncology patients. Anesth Analg, 101(6):1623-7.

Apfelbaum JL, Chen C, Mehta SS, Gan TJ. 2003. Postoperative pain experience: results from a national survey suggest postoperative pain continues to be undermanaged. Anesth Analg, 97(2):534-40.

Ashburn MA, Streisand J, Zhang J, et al. 1995. The iontophoresis of fentanyl citrate in humans. Anesthesiology, 82(5):1146-53.

Bonnet F, Eberhart L, Wennberg E. 2006. Fentanyl HCL iontophoretic transdermal system (ITS) versus intravenous patient-controlled analgesia (IV-PCA): Staff time savings [poster]. Fifth Congress of the European Federation of IASP, September 2006, Istanbul, Turkey.

Camu F, Van Aken H, Bovill JG. 1998. Postoperative analgesic effects of three demand-dose sizes of fentanyl administered by patient-controlled analgesia. Anesth Analg, 87(4):890-5.

Chelly JE, Grass J, Houseman TW, et al. 2004. The safety and efficacy of a fentanyl patient-controlled transdermal system for acute postoperative analgesia: a multicenter, placebo-controlled trial. Anesth Analg, 98(2):427-33.

Chelly JE. 2005. An iontophoretic, fentanyl $\mathrm{HCl}$ patient-controlled transdermal system for acute postoperative pain management. Expert Opin Pharmacother, 6(7):1205-14.

Curdy C, Kalia YN, Guy RH. 2001. Non-invasive assessment of the effects of iontophoresis on human skin in-vivo. J Pharm Pharmacol, 53(6):769-77.

Dolin SJ, Cashman JN, Bland JM. 2002. Effectiveness of acute postoperative pain management: I. Evidence from published data. $\mathrm{Br} J$ Anaesth, 89(3):409-23.

Dunwoody CJ, Krenzischek DA, Pasero C, et al. 2008. Assessment, physiological monitoring, and consequences of inadequately treated acute pain. Pain Manag Nurs, 9(1 Suppl):S11-21.

Fearon KC, Ljungqvist O, Von Meyenfeldt M, et al. 2005. Enhanced recovery after surgery: a consensus review of clinical care for patients undergoing colonic resection. Clin Nutr, 24(3):466-77.

Grond S, Hall J, Spacek A, et al. 2007. Iontophoretic transdermal system using fentanyl compared with patient-controlled intravenous analgesia using morphine for postoperative pain management. $\mathrm{Br} J$ Anaesth, 98(6):806-15.

Grond S, Radbruch L, Lehmann KA. 2000. Clinical pharmacokinetics of transdermal opioids: focus on transdermal fentanyl. Clin Pharmacokinet, 38(1):59-89.

Harding G, Vallow S, Leidy NK, et al. 2007. Ease of care with patient controlled analgesia systems: questionnaire development and validation. $J$ Adv Nurs, 59(5):530-41

Hartrick CT, Bourne MH, Gargiulo K, et al. 2006. Fentanyl iontophoretic transdermal system for acute-pain management after orthopedic surgery: a comparative study with morphine intravenous patient-controlled analgesia. Reg Anesth Pain Med, 31(6):546-54.

Herndon CM. 2007. Iontophoretic drug delivery system: focus on fentanyl. Pharmacotherapy, 27(5):745-54.
Joshi GP, Ogunnaike BO. 2005. Consequences of inadequate postoperative pain relief and chronic persistent postoperative pain. Anesthesiol Clin North America, 23(1):21-36.

Kalia YN, Naik A, Garrison J, Guy RH. 2004. Iontophoretic drug delivery. Adv Drug Deliv Rev, 56:619-58.

Kehlet H. 1997. Multimodal approach to control postoperative pathophysiology and rehabilitation. Br J Anaesth, 78(5):606-17.

Macintyre PE. 2001. Safety and efficacy of patient-controlled analgesia. Br J Anaesth, 87(1):36-46.

Minkowitz HS, Rathmell JP, Vallow S, et al. 2007. Efficacy and safety of the fentanyl iontophoretic transdermal system (ITS) and intravenous patient-controlled analgesia (IV PCA) with morphine for pain management following abdominal or pelvic surgery. Pain Med, 8(8):657-68.

Muijsers RB, Wagstaff AJ. 2001. Transdermal fentanyl: an updated review of its pharmacological properties and therapeutic efficacy in chronic cancer pain control. Drugs, 61(15):2289-307.

Panchal SJ, Damaraju CV, Nelson WW, et al. 2007. System-related events and analgesic gaps during postoperative pain management with the fentanyl iontophoretic transdermal system and morphine intravenous patient-controlled analgesia. Anesth Analg, 105(5):1437-41.

Pikal MJ. 2001. The role of electroosmotic flow in transdermal iontophoresis. Adv Drug Deliv Rev, 46(1-3):281-305.

Practice guidelines for acute pain management in the perioperative setting: an updated report by the American Society of Anesthesiologists Task Force on Acute Pain Management. 2004. Anesthesiology, 100(6):1573-81.

Sathyan G, Jaskowiak J, Evashenk M, et al. 2005a. Characterisation of the pharmacokinetics of the fentanyl $\mathrm{HCl}$ patient-controlled transdermal system (PCTS): effect of current magnitude and multiple-day dosing and comparison with IV fentanyl administration. Clin Pharmacokinet, 44(Suppl 1):7-15.

Sathyan G, Zomorodi K, Gidwani S, et al. 2005b. The effect of dosing frequency on the pharmacokinetics of a fentanyl $\mathrm{HCl}$ patient-controlled transdermal system (PCTS). Clin Pharmacokinet, 44(Suppl 1):17-24.

Sidebotham D, Dijkhuizen MR, Schug SA. 1997. The safety and utilization of patient-controlled analgesia. J Pain Symptom Manage, 14(4):202-9

Tramer MR, Walder B. 1999. Efficacy and adverse effects of prophylactic antiemetics during patient-controlled analgesia therapy: a quantitative systematic review. Anesth Analg, 88(6):1354-61.

Varvel JR, Shafer SL, Hwang SS, et al. 1989. Absorption characteristics of transdermally administered fentanyl. Anesthesiology, 70(6):928-34.

Vicente KJ, Kada-Bekhaled K, Hillel G, et al. 2003. Programming errors contribute to death from patient-controlled analgesia: case report and estimate of probability. Can J Anaesth, 50(4):328-32.

Viscusi ER, Reynolds L, Chung F, et al. 2004. Patient-controlled transdermal fentanyl hydrochloride vs intravenous morphine pump for postoperative pain: a randomized controlled trial. JAMA, 291(11):1333-41.

Viscusi ER, Reynolds L, Tait S, et al. 2006. An iontophoretic fentanyl patient-activated analgesic delivery system for postoperative pain: a double-blind, placebo-controlled trial. Anesth Analg, 102(1):188-94.

Walder B, Schafer M, Henzi I, et al. 2001. Efficacy and safety of patient-controlled opioid analgesia for acute postoperative pain. A quantitative systematic review. Acta Anaesthesiol Scand, 45(7):795-804. 
